\title{
Endoscopic and histological staging: Deciding factors in the treatment of chronic rhinosinusitis
}

\author{
Amalia Neagu, Codrut Sarafoleanu \\ ENT\&HNS Department, "Sfanta Maria" Hospital, Bucharest, Romania
}

\section{ABSTRACT}

Chronic rhinosinusitis represents an inflammatory condition of the nose and paranasal sinuses, characterized by two or more symptoms. The diagnosis of acute rhinosinusitis is made, most often, clinically; however, in the case of chronic rhinosinusitis, imaging, bacteriological, as well as the histological investigation are necessary in order to establish the diagnosis and to exclude other diseases that have caused and/or are associated with rhinosinusitis. Different scores (clinical, imagistic) have been described over time, being considered as important factors in the therapeutic decision (surgical or conservative) in patients with chronic rhinosinusitis. The authors present their experience in using and relying on endoscopic and histological scores.

KEYWORDS: chronic rhinosinusitis, endoscopic score, histological score, polyps, treatment.

\section{INTRODUCTION}

Chronic rhinosinusitis represents an inflammatory condition of the nose and paranasal sinuses, characterized by two or more symptoms, of which one should be obstruction or nasal congestion or nasal secretion (anterior or posterior rhinorrhea), accompanied or not by facial pain or facial pressure feeling, with or without reduction or loss of smell, symptoms lasting more than 12 weeks. The endoscopic examination describes either polyps and/or mucopurulent rhinorrhea from the middle meatus and/or oedema or congestion of the mucosa from the middle meatus and/ or CT changes in the mucosa from the ostiomeatal complex and/or of the sinus mucosa ${ }^{1}$.

Causes of rhinosinusitis are multiple and include:

- allergic causes (most commonly)

- infectious

- aspirin intolerance

- non-allergic rhinosinusitis with eosinophilia (NARES)

- idiopathic

- occupational / by exposure to environmental factors

- systemic diseases (Wegener's granulomatosis, vasculitides)

- mechanical or structural causes (nasal septum deviation, neoplasms, immotile cilia syndrome)

- drug-induced (topical / systemic: propranolol, oral contraceptives, reserpine, nasal sprays)

- pregnancy (through the combined effect of pregnancy hormones and increased blood volume)
The diagnosis of acute rhinosinusitis is made, most often, clinically; however, in the case of chronic rhinosinusitis, imaging, bacteriological, as well as the histological investigation are necessary in order to establish the diagnosis and to exclude other diseases that have caused and/or are associated with rhinosinusitis.

Treatment of rhinosinusitis includes medical and surgical therapeutic methods, depending on the etiology, degree of lesions, complications and impaired quality of life.

\section{STAGING SYSTEMS IN RHINOSINUSITIS}

Different scores (clinical, imagistic) have been described over time, being considered as important factors in the therapeutic decision (surgical or conservative) in patients with chronic rhinosinusitis.

The severity of the disease can be divided, depending on the clinical score, in mild, moderate and severe $^{2,3}$. This classification is subjective and is realized based on patients' response to the question: how annoying are the symptoms of rhinosinusitis? The patient will answer by ticking on a straight-line segment, $10 \mathrm{~cm}$ long, labelled "not annoying" in one end and "very annoying" in the other. Once noted, the response is measured on the visual analogue scale (VAS):

- Mild: VAS = 0-3

- Moderate: VAS >3-7

- Severe: VAS >7-10

A subjective clinical score above 5 is translated by affecting the patient's quality of life. 
The need for a quantitative, objective quantification of the rhinosinusal damage, as well as of the response to treatment, led to the emergence of computed-tomographic scores, the most commonly used being the Lund-Mackay score (Table 1$)^{4}$.

Calculation of the radiological score $(\mathrm{CT})^{47}$ is made preoperatively, taking into account the degree of sinus opacification, as well as the permeability of the ostiomeatal complex (COM) (free/obstructed):

1. According to the degree of opacification

- Sinus with normal appearance $=0$ points

- Partially opacified sinus $=1$ point

- Completely opacified sinus $=2$ points

2. According to $\mathbf{C O M}$

- Free ostiomeatal complex $=0$ points

- Blocked ostiomeatal complex $=2$ points

Nevertheless, although this method of quantification was received with enthusiasm at the beginning, several studies have shown that the extension of lesions on the sinus CT scanning (Lund-Mackay) ${ }^{4}$ do not correlate with the quality of life scores; in other words, the degree of rhinosinusal damage radiologically detected is inconsistent with patients' symptoms ${ }^{5,6}$.

Nasal and rhinosinusal endoscopic examination represents another method for assessing the extent of the lesions found in chronic rhinosinusitis. Using the nasal endoscopic examination, Lund and Mackay have created an endoscopic score ${ }^{4}$, which takes into account the presence or absence of the following elements: polyps, oedema, crusts, synechiae, secretions. A score is given for each parameter taken into consideration and for each nasal passage, as follows:

1. Polyps: 0 points (absent), 1 point (present in the middle meatus), 2 points (exceed the middle meatus);

2. Oedema, crusts, synechiae: 0 points (absent), 1 point (moderate), 2 points (severe);

3. Secretions: 0 points (absent), 1 point (serous, transparent), 2 points (mucous, purulent).

The minimum score that can be obtained by adding these scores, on both nasal passages, is 0 , and the maximum one 20.

According to the experience of the ENT Depart- ment of "Sfanta Maria" Hospital, Bucharest, the aspect of the endosinusal mucosa revealed by the sinusoscopy may have predictive value in the treatment of chronic rhinosinusitis ${ }^{8}$. Thus, 5 types of maxillary endosinusal aspects are recognized (Figure 1.a-d):

- Type 0: normal, transparent mucosa, without secretions, with a vascular pattern within normal limits - in this case, the mucociliary and ostial functions are normal;

- Type 1: mucosa with emphasized vascular pattern, slightly more "opaque", with serous secretions (Figure 1a) - requires drug treatment to restore the mucociliary and ostial functions;

- Type 2: thickened mucosa, with more consistent secretions, with heavily emphasized vascular pattern, cystic dilatation of submucosal glands and changes in the chorion (Figure 1b) - respond to medication or endoscopic surgery of repermeabilization of ostial transitional areas;

- Type 3: important changes in the mucosa, polyps, cysts, mucopurulent secretions (Figure 1c) - requires endoscopic surgical treatment to restore rhinosinusal ventilation, followed by adjunctive medical therapy;

- Type 4: mucosal hyperplasia and metaplasia, polyps, fungosities and caseum (Figure 1d) - denotes a compromised structural and functional endosinusal mucosa, frequently necessitating Caldwell-Luc type radical surgery.

Since the assessment of endoscopic aspects involves a certain degree of subjectivity, in the "Sfanta Maria" ENT Department, a correlation has been made between the endoscopic score and a histopathological score that objectively appreciates the structural changes in the maxillary sinus mucosa, taking into account the following parameters:

- the density and distribution of the inflammatory infiltrate

- the presence of eosinophils

- intramucosal oedema presence

- the presence of neoformed vessels (neoangiogenesis)

- fibrosis

Table 1

The Lund-Mackay CT staging ${ }^{4}$

\begin{tabular}{cc}
\hline Sinus & Right side score \\
\hline Maxillary $(0,1,2)$ & \\
\hline Anterior ethmoid $(0,1,2)$ \\
\hline Posterior ethmoid $(0,1,2)$ \\
\hline Frontal $(0,1,2)$ \\
\hline Sphenoid $(0,1,2)$ \\
\hline Ostiomeatal complex $(0$ or 2$)$ \\
\hline Total points
\end{tabular}



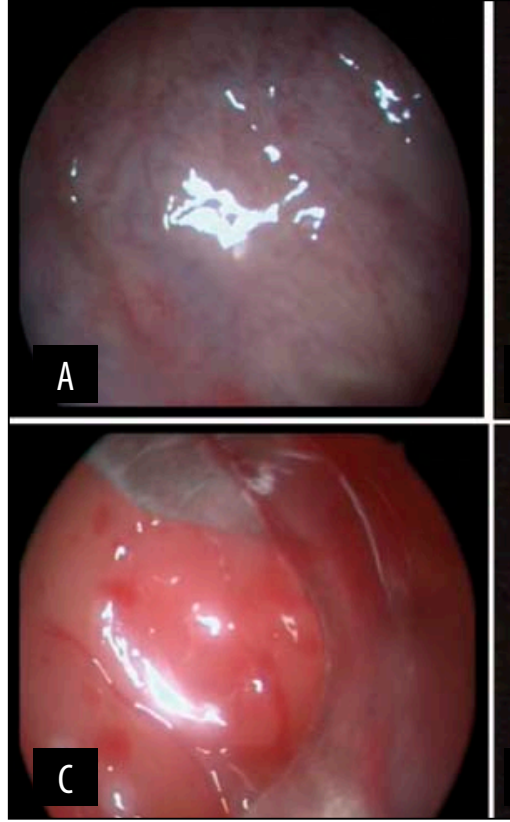

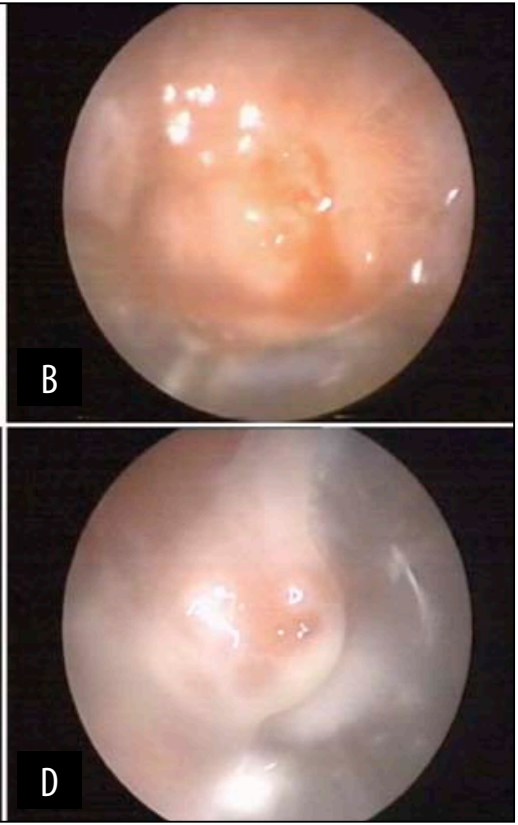

Figure 1 Sinusoscopy aspects of the maxillary sinus mucosa (A) mucosa with emphasized vascular pattern, slightly more "opaque", with serous secretions; (B) thickened mucosa, with more consistent secretions, with heavily emphasized vascular pattern, cystic dilatation of submucosal glands and changes in the chorion; (C) important changes in the mucosa, polyps, cysts, mucopurulent secretions; (D) mucosal hyperplasia and metaplasia, polyps, fungosities and caseum
This score is obtained by the histopathological exam of some fragments of maxillary sinus mucosa, collected prior to the surgery itself (by sinusoscopy). After the histopathological exam, a score is given for each parameter taken into account, and the total score represents the histopathological score (Table 2$)^{9}$.

After analyzing each parameter and granting the appropriate score, a total score ranging from 0 to 11 points has been revealed. A division of anatomopathological changes into four degrees has been made:

Grade I - Normal score (between 0 and 3 points) = Without changes/ Minimal damage

Grade II - Low score (between 4 and 7 points) = Mild damage

Grade III - Average score (between 8 and 10 points) = Moderate damage

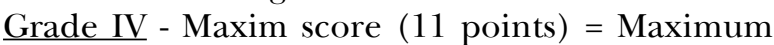
damage

The scores obtained have been used in determining the therapeutic conduct, as follows:

- Score 0-3, fibrosis 0 - conservative treatment and endoscopic follow-up

- Score 3-7, fibrosis 1 - functional endoscopic sinus surgery - FESS

- Score 7-10, fibrosis 2 - extended endoscopic sinus surgery - ESS

- Score 11, fibrosis 3 - classical surgery - maxillary sinus radical Caldwell-Luc cure

The histological score of mucosal lesions can be used in the therapeutic decision and as a prognostic factor for the therapeutic success in chronic rhinosinusitis.

In our clinic, a prospective study was made regarding the correlation between the endoscopic score and the histopathological one and their use in the therapeutic decision in chronic maxillary rhinosinusitis. Excellent results have been obtained in the study - the therapeutic success rate, when used, has been above $90 \%$, the number of radical surgical cures has decreased and there have been cases medically treated with very good results ${ }^{9}$.

Starting from this study, which has validated the two scores and showed their value in the therapeutic decision (endoscopic surgery versus classical surgery in chronic maxillary rhinosinusitis), we have decided to extend its use in those cases with multiple sinuses involvement.

Therefore, we have started from the fact that all sinus cavities are coated with ciliated cylindrical pseudostratified epithelial tissue, structurally similar to the nasal mucosa (Figure 2); the sinus mucosa is thinner and less vascularized than that of the nasal fossae, also presenting a fibrous layer adjacent to the periosteum.

Due to the structural unit of the nasal mucosa and of the paranasal sinuses, there is currently a consensus in terms of replacing the term sinusitis with rhinosinusitis, because inflammation of the sinus mucosa is preceded and/or concomitant with that of the nasal mu-



Figure 2 Ciliated respiratory epithelium, separated from the submucosa by a basement membrane; the seromucous glands can be observed in the submucosa 
Table 2

Rhinosinusal histopathological score ${ }^{9}$

\begin{tabular}{crc}
\hline Histopathological parameter & Score & Histopathological Image \\
\hline $\begin{array}{c}\text { 1. Density and distribution of the in- } \\
\text { flammatory infiltrate }\end{array}$ & -1 point - rare lymphatic elements, diffusely distributed \\
\hline & -2 points - frequent lymphatic elements, diffusely distributed \\
\hline & -3 points - frequent lymphatic elements, subepithelial $+/-$ peri-
\end{tabular}

2. Presence of eosinophils

-0 points - absent

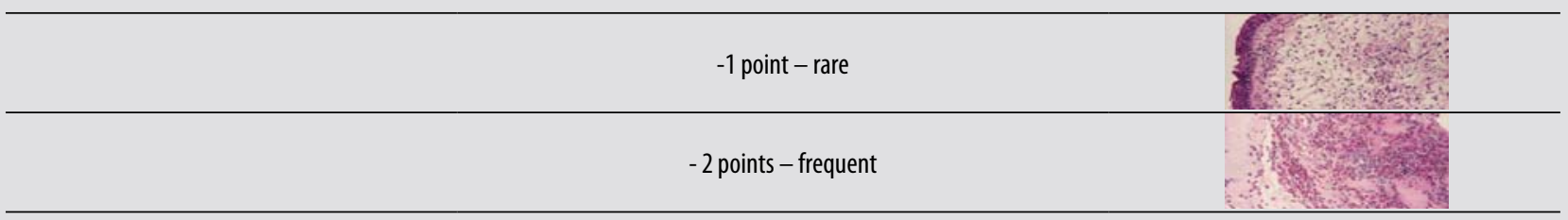

3. Intramucosal oedema presence

- 0 points - without oedema

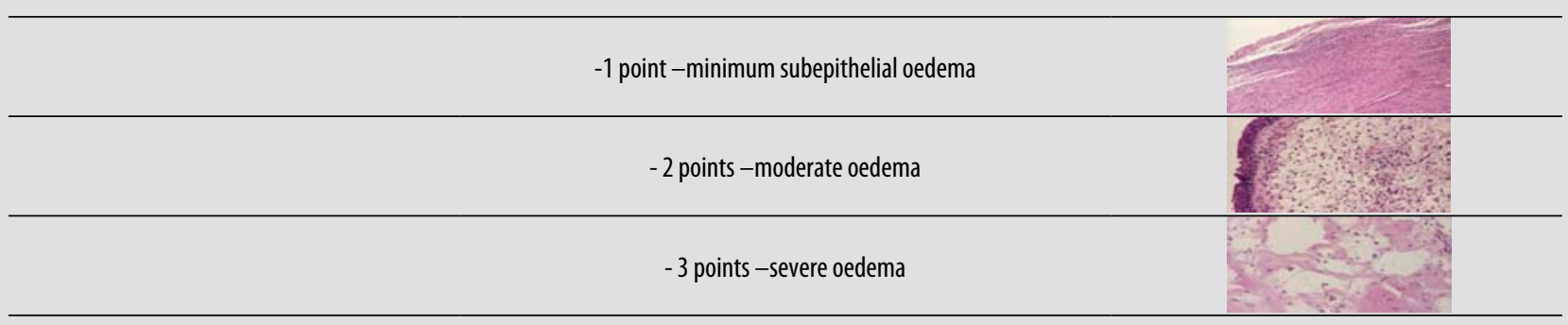

4. The presence of neoformation vessels

- 1 point - a minimum of neoformed vessels

\begin{tabular}{c}
\hline-2 points - frequent neoformed vessels \\
\hline-3 points - frequent neoformed vessels with thickened walls due \\
to fibrosis (in the walls and perivascularly)
\end{tabular}

5. Intramucosal oedema presence

-0 points - absent fibrosis

-1 point - minimal fibrosis, periglandular $+/$ - intramucosal

- 2 points - moderate, with cystic glandular dilatations

- 3 points - marked, with irreversible mucosal hyperplasia

cosa; in other words, rhinitis and sinusitis coexist ${ }^{1,10}$. Consequently, the anatomopathological changes in the nasal mucosa should be similar to those in the sinus mucosa (excluding odontogenic sinusitis).

As a result, in order to expand the anatomopathological score in other sinus disorders too, not only that of the maxillary sinus, and for a better patient compliance in determining the anatomopathological score, we have studied a group of 44 patients with chronic rhino-pansinusitis, in whom we have proved the equivalence of the sinus mucosa biopsy (maxillary, ethmoidal) to that in the middle nasal turbinate. Comparative anatomopathological examinations of the biopsy fragments collected in each patient from the sinus and, respectively, from the middle turbinate, have been performed; moreover, all previously described param- 
eters have been analyzed, and the histopathological scores obtained independently have overlapped in a $90 \%$ proportion (statistically significant).

The histological score is to be completed in the future by studying the proteins in the respiratory mucosa, which participates in regulating the local immunity and/or the local inflammation.

Therefore, in our clinic, a research study has been carried out, in collaboration with the Faculty of Medicine of the University of Siena, in order to identify how the protein HMGB1 (High Mobility Group Box 1) influences the inflammation in chronic rhinosinusitis with/without nasal polyposis ${ }^{11,12}$.

In the ENT field, there are few works dealing with the importance of this cytokine; the first of them appeared in 2012 and investigates the role of this protein in the rhinosinusal inflammatory pathology ${ }^{13,14}$.

Recently, the importance of the protein HMGB1 in the pathogenesis of many inflammatory diseases has been proved. This protein is released from necrotic cells or from the cells of the activated immune system (activated macrophages or monocytes) and acts on the specific receptors in the cell membrane, causing the release of proinflammatory mediators, endothelial activation and the survival of inflammatory cells, especially of eosinophils ${ }^{11}$. The role of cytokine of HMGB1 is exercised when released in the extracellular environment; this localization is a negative prognostic marker, as highlighted in our study too. The discovery of HMGB1 protein in the nasal secretion of patients with allergic and non-allergic inflammatory diseases of the nose and the paranasal sinuses may contribute to the chronicization of the inflammatory process. As a consequence, the inhibition of the HMGB1 protein may be an effective therapeutic strategy in rhinosinusal chronic inflammation.

Subsequently, we have continued the research of inflammatory mechanisms, by participating in a study that aimed at determining the role of the PLUNC protein (palate, lung and nasal epithelium clone protein) in the inflammation etiopathogenesis in the ENT sphere.

Therefore, in the fragments collected from the middle nasal turbinate mucosa, we have also searched for the presence of the PLUNC protein, a protein specific of the upper airways, which participates in local defence by a bacteriological effect and physicochemical effects ${ }^{15,16}$. It has been proved that it is produced by neutrophils after bacterial stimulation and it is assumed that low expression of this protein may contribute to the pathogenic mechanism of chronic rhinosinusitis.

\section{CONCLUSIONS}

The histological score indicates the degree of structural changes in the rhinosinusal mucosa and its capacity for regeneration.
The histopathological score obtained by examining the sinus mucosa fragments (ethmoidal or maxillary) is superimposable to that obtained by examining the fragments collected from the middle nasal turbinate mucosa.

The endoscopic and the histological scores are reliable indicators for the type of treatment that needs to be applied to patients with chronic rhinosinusitis.

From previous studies conducted in our clinic, there is a correlation between the histopathological score and the endoscopic one and they may determine the type of treatment indicated with a very high success rate (above $90 \%$ ).

\section{REFERENCES}

1. Fokkens W.J., Lund V.J., Mullol J., Bachert C., Alobid I., Baroody F., Cohen N., Cervin A., Douglas R., Gevaert P., Georgalas C., Goossens H., Harvey R., Hellings P., Hopkins C., Jones N., Joos G., Kalogjera L., Kern B., Kowalski M., Price D., Riechelmann H., Schlosser R., Senior B., Thomas M., Toskala E., Voegels R., Wang de Y., Wormald P.J. - EPOS 2012: European position paper on rhinosinusitis and nasal polyps 2012. A summary for otorhinolaryngologists. Rhinology, 2012;50(1):1-12.

2. Naidoo Y., Tan N., Singhal D., Wormald P.J. - Chronic rhinosinusitis assessment using the Adelaide Disease Severity Score. J Laryngol Otol., 2013;127(Suppl 2):S24-8.

3. Rizk H.G., Ferguson B.J. - Categorizing Nasal Polyps by Severity and Controller Therapy. Arch Otolaryngol Head Neck Surg., 2012;138(9):846-53.

4. Lund V.J., Mackay I.S. - Staging in rhinosinusitis. Rhinology, 1993;107:183-4.

5. Moghadasi H., et al. - Association bBetween Clinical Symptoms and CT Findings in Chronic Rhinosinusitis. Iran J Radiol., 2008;5(3):145-150.

6. Bradley D.T., Kountakis S.E. - Correlation between computed tomography scores and symptomatic improvement after endoscopic sinus surgery. Laryngoscope, 2005;115(3):466-9.

7. Hopkins C., Browne J.P., Slack R., Lund V.J., Brown P. - The Lund-Mackay staging system for chronic rhinosinusitis: How is it used and what does it predict? Otolaryngol Head Neck Surg., 2007;137(4):555-61.

8. Sarafoleanu C. - Metode de explorare paraclinica si functionala utilizate in suferintele rino-sinusale. In: Sarafoleanu C, eds. - Rinologie. Bucharest: Editura Medicala, 2003;p.83-127.

9. Manea C., Iosif C., Sarafoleanu D. - The value of histopathologic score as predictive factor in choosing the optimal surgical treatment for chronic rhinosinusitis. Romanian Journal of Rhinology, 2012;2(6):90-95.

10. Benninger M.S., Ferguson B.J., Hadley J.A., et al. - Adult chronic rhinosinusitis: definitions, diagnosis, epidemiology, and pathophysiology. Otolaryngol Head Neck Surg., 2003;129(3 Suppl):S1-32.

11. Iosif C., Jianu E., Sarafoleanu C., Duda R., Panaitescu E. - The role of HMGB1 protein in chronic rhinosinusitis with nazal polyposis - is it a real proinflammatory mediator? Romanian Journal of Rhinology, 2013;3(10):71-80.

12. Fransson M., Benson M., Wennergren G., Cardell L.O. - A role for neutrophils in intermittent allergic rhinitis. Acta Otolaryngol., 2004 Jun;124(5):616-620.

13. Bellussi L.M., Chen L., Chen D., Passali F.M., Passali D. - The role of high mobility group box 1 chromosomal protein in the pathogenesis of chronic sinusitis and nasal polyposis Acta Otorhinolaryngol Ital., 2012;32:386-392.

14. Bellussi L.M., Iosif C., Sarafoleanu C., Jianu E., Duda R., Panaitescu E., Passali F.M., Passali D. - Are HMGB1 protein expression and secretion markers of upper airways inflammatory diseases? J Biol Regul Homeost Agents, 2013 JulSep;27(3):791-804

15. Bartlett J.A., Gakhar L., Penterman J., Singh P., Mallampalli R.K., Porter E., McCray P.B. Jr. - PLUNC: a multifunctional surfactant of the airways Biochemical Society Transactions, 2011;39(4):1012 - 1016.

16. Bartlett J.A., Hicks B.J., Schlomann J.M., Ramachandran S., Nauseef W.M., McCray P.B. Jr. - PLUNC is a secreted product of neutrophil granules. J Leukoc Biol., 2008;83(5):1201-1206. 